\title{
CRISI E RINASCITA DELL'EUROPA: ECHI DEL DIBATTITO FENOMENOLOGICO
}

\author{
Michele Lenoci ${ }^{I}$
}

RIASSUNTO: Il saggio, attraverso un'analisi delle riflessioni di Husserl e Scheler, si propone di esaminare la loro concezione sull'Europa e la tradizione culturale europea, mettendo in rilievo l'evoluzione e lo sviluppo delle loro posizioni avanti e dopo la prima Guerra mondiale.

PAROLE CHIAVE: Europa e filosofia. Fenomenologia. Husserl. Scheler. Prima guerra mondiale.

Nel dibattito novecentesco ritorna frequente il tema del rapporto tra la filosofia e l'Europa: la sua crisi, le cause della decadenza, la sua possibile rinascita e il suo destino; in esso si intrecciano e si mescolano prospettive di varia natura e criteri di giudizio assai differenti e fra loro spesso incompatibili; così come a considerazioni più strettamente e immediatamente politiche si alternano analisi che proiettano la storia europea sullo sfondo, più ampio e globale, della vicenda dell'Occidente, intesa come affermazione e variazioni della razionalità o come manifestazione e dimenticanza dell'Essere, cosicché i registri impiegati coinvolgono, di volta in volta, l'ontologia, la natura della filosofia, il rapporto con la scienza e la tecnica, i risultati del lungo cammino della modernità. E quanto più le considerazioni si fanno epocali e abbracciano, con un solo colpo d'occhio, interi secoli, tanto più l'analisi rischia la genericità o conclusioni troppo facilmente condivisibili, perché assai prossime alla tautologia. Nel secolo scorso, lungo e tormentato, sullo scenario europeo si sono avvicendati eventi imponenti e talora tragici, così come crisi limitate e contingenti, ma pur sempre capaci di inserire nuove variabili e sfumature, i quali tutti fanno da sfondo a quelle considerazioni e offrono loro ampio materiale da esaminare: lo spartiacque costituito dalla prima guerra mondiale; la grande depressione

\footnotetext{
${ }^{1}$ Professore ordinario di Storia della Filosofia contemporanea. Preside della Facoltà di Scienze della formazione. Università Cattolica del Sacro Cuore Milano. Presidente della Società Italiana di Storia della Filosofia.michele.lenoci@unicatt.it
} 
post-bellica, che conferma ed enfatizza il senso di scacco e decadenza, così come pare attestare il fallimento dell'idea moderna di progresso, ritenuto inarrestabile almeno sino agli inizi del secolo XX, e accrescere la sfiducia nelle capacità definitivamente risolutive della tecnicizzazione del mondo; l'epoca dei totalitarismi, di diversa natura, ma egualmente oppressivi e capaci di causare lo sterminio di milioni di persone con differenti pretesti o motivazioni ideologiche; la seconda guerra mondiale, con il suo carico di lutti e con il lungo travaglio politico, morale, religioso, seguito alla terribile pagina della Shoah; la divisione rispetto all'Europa orientale e la convinzione di una realtà europea divisa dalla cortina di ferro, e perciò mutilata e incompiuta, mentre le speranze utopistiche suscitate da esperimenti rivoluzionari, miranti a creare l'uomo nuovo in società senza sfruttamento, andavano deluse e il loro fallimento produceva effetti di lunga durata su tutto il continente, che hanno allungato la loro ombra sino al 1989 e ben oltre; le realizzazioni comunitarie in campo economico e politico, sino all'adozione della moneta unica e alla riunificazione della Germania, tornata a essere decisivo punto di equilibrio e snodo essenziale nelle questioni europee.

Tutti questi fatti, i cui effetti hanno spesso prodotte ferite non ancora rimarginate e reazioni non metabolizzate, che impediscono una considerazione prospettica sufficientemente obbiettiva e distaccata, hanno determinato valutazioni di opposta natura, sia nel corso del secolo, sia alla sua conclusione, in sede di bilancio: prevalenti sono i toni pessimistici, giacché, pur non dimenticando i passi in avanti e i cambiamenti in senso democratico e umanistico, compiuti soprattutto nel secondo dopoguerra, così come il netto miglioramento del complessivo tenore di vita, anche grazie ai legami sempre più stretti imposti dalla cooperazione internazionale, il senso di disorientamento e la perdita di un centro rendono spesso l'Europa divisa sulle decisioni da prendere, tentata dalla frammentazione e dai particolarismi, incapace di precisare la sua identità, cioè quegli elementi costitutivi, e condivisi, cui fare appello per giustificare sacrifici o per attingere un orientamento unitario e indicare una meta comune per il futuro. Si lamenta che ai successi in campo economico non abbia fatto riscontro un consolidamento in campo politico, e questa carenza vien anche attribuita a un'attenuata consapevolezza culturale, alla mancanza di visione aperta al futuro e piena di fiducia. La stessa polemica sulle radici dell'Europa e le discussioni quando si è cercato di identificarle o fissarle secondo scale di priorità, prima ancora che contrapposizioni ideologiche, denunciano un disagio e rivelano un'esigenza intellettuale e 
critica, che il passare dei decenni e l'avvento del nuovo millennio non hanno ancor pienamente soddisfatto, né adeguatamente affrontato.

Si tratta di uno stato d'animo che al fondo riecheggia, sia pure con ovvie differenze rilevanti, le impressioni provate, tra Cinque e Settecento, da parte dei dotti e dalla Repubblica delle Lettere, di fronte a civiltà diverse da quella europea o all'iniziale contatto con i popoli primitivi: nostalgia verso un'ingenuità ormai perduta e smarrimento perché dal confronto con altri paesi per molti aspetti si poteva anche uscire sconfitti o ridimensionati nelle proprie pretese. Ma, allora, nonostante valutazioni contrastanti, dovute agli echi ancora vivaci delle guerre di religione o alle polemiche contro l'assolutismo, permanevano alcuni punti fermi, capaci di alimentare un certo orgoglio europeo e un solido ottimismo verso il futuro: l'esaltazione delle scienze e delle arti, della tecnica e dei progressi dello spirito umano, così come il senso della libertà e l'elogio della ragione caratterizzano un corpus culturale e morale, uno stile di vita sociale e di atteggiamenti, che danno costante unità al sentimento europeo e delineano una peculiarità, se non un primato.

E tuttavia lo stato d'animo, nelle vicende del secolo scorso, appare per molti aspetti assai diverso: due guerre mondiali sono nate in Europa a causa di conflitti tra europei, i processi di decolonizzazione hanno messo in luce le violenze perpetrate in secoli di oppressione di molti popoli extra-europei, i totalitarismi, i campi di sterminio, la contrapposizione tra i due lati della cortina di ferro sembrano aver esaurito le risorse spirituali di un continente, che, dopo aver perduto la centralità economica e politica, sente minacciato anche il suo primato culturale e umanistico, o, forse, di quel patrimonio tende a dimenticare la portata e il valore, senza più vedere in esso il nucleo della propria identità, certamente plurale, ma non indeterminata e indistinta. Sembra quasi che la cosiddetta fine della modernità e la polemica contro l'indebita estensione di modelli di interpretazione scientifica ad ambiti non pertinenti, induca a cedere a una deriva irrazionalistica, in cui i riferimenti tradizionali della cultura europea si dissolvono in un prospettivismo illimitato e in un pragmatistico elogio dei compromessi fine a se stessi, sui quali diventa poi difficile delineare un progetto per il futuro.

In questo ampio contesto i filosofi hanno fatto sentire, in momenti e con accenti diversi, la loro voce, collocando le osservazioni sull'Europa all'interno del loro itinerario di pensiero, quasi a trovare per esso una conferma e un'esemplificazione fattuale; d'altro lato, la situazione storica concreta, in cui ciascuno è collocato, modula e determina un orizzonte che 
inevitabilmente influenza i caratteri e le variazioni dei diversi percorsi teorici. Piuttosto che indugiare su una panoramica ad ampio raggio, per trarre un forse impossibile bilancio di tanto differenziato impegno, nel corso di queste pagine, isolando un momento ben delimitato, preferisco soffermarmi su alcuni echi abbastanza connessi, significativi e coerenti, che possono offrire un esempio della confluenza nel dibattito di molteplici componenti, atte a renderlo più complesso, non privo di ambiguità e aperto a esiti differenziati e talora contraddittori. Mi riferisco alle posizioni assunte da due esponenti rilevanti della scuola fenomenologica tedesca - Edmund Husserl e Max Scheler - negli anni che vanno dalla vigilia della prima guerra mondiale sin quasi alle soglie della seconda: esse sono significative, in quanto rivelano un panorama denso di luci e ombre, risentono di condizionamenti ampiamente condivisi tra gli intellettuali dell'epoca e delineano esiti assai sintomatici.

\section{AgLI INIZI DELLA PRIMA GUERRA MONDIALE}

Come è noto, alla vigilia della prima guerra mondiale il nazionalismo e l'imperialismo sono diventati ideologia capace di agitare le masse, non solo in Germania ma in tutta Europa; persino i partiti socialisti e le sinistre, con esclusione di quelli italiani, emarginano le tendenze pacifiste e i richiami internazionalisti e nella loro grande maggioranza sostengono le posizioni patriottiche dei rispettivi governi. Soltanto a guerra conclusa la dolorosa constatazione degli esiti drammatici prenderà il posto dell' effervescenza retorica precedente e cercherà, soprattutto in terra tedesca, le vie di una riconciliazione e di una rinascita. Ma nel 1914 i fronti contrapposti mobilitano anche gli intellettuali per un impegno, ora, allo scopo di diffondere la democrazia e la libertà, ora, a salvaguardia di una tradizione culturale e spirituale, che si vede minacciata dal prevalere di una mentalità utilitaristica, tecnica ed egualitaria. Mentre desiderio della Germania, diventata una grande potenza economica e politica, è quello di assumere un ruolo coerente e adeguato anche sulla scena mondiale, gli intellettuali tendono a sottolineare il carattere culturale ed essenzialmente difensivo dell'impresa bellica, anche quando le apparenze rivelano innegabile spirito aggressivo ${ }^{2}$. Se le forze dell'Intesa si mobilitano contro il militarismo prussiano e il dispotismo russo, di stampo ancora medioevale, in Germania quasi tutti i professori universitari, guidati da von Wilamowitz-Möllendorf, e un folto gruppo di intellettuali si associano ai

${ }^{2} \mathrm{Si} \mathrm{veda,} \mathrm{sempre} \mathrm{pertinente:} \mathrm{FISCHER,} \mathrm{F.} \mathrm{Assalto} \mathrm{al} \mathrm{potere} \mathrm{mondiale.} \mathrm{La} \mathrm{Germania} \mathrm{nella} \mathrm{guerra} \mathrm{1914-}$ 1918. Torino: Einaudi, 1965. 
rappresentanti delle forze economiche e sociali tra il 1914 e il 1915 non tanto per avanzare pretese di ampliamenti territoriali, ma per sottolineare come questa guerra costituisca un dovere e un impegno vitale e culturale, in difesa del diritto di un popolo - quello tedesco - a esistere e ad operare e a tutela della cultura, spirituale e ideale, di cui ci si considerano custodi e difensori. Per capire questo stato d'animo, basti pensare all'intensa coltivazione della cultura classica, storica e filologica, esplicitamente mirante a far rivivere in terra tedesca gli ideali spirituali e civili dell'antica Grecia, in modo che potessero formare non tanto un ristretto numero di eruditi, ma la mentalità di quanti avrebbero costituito la spina dorsale amministrativa del nuovo stato prussiano.

In questa prospettiva non può trovare ascolto l'offerta della libertà e della democrazia rivolta alla Germania, oppressa da un militarismo non democratico $^{3}$; anzi vengono richiamati precedenti storici assai significativi: la lotta di Federico II contro la grande coalizione degli stati europei, che non intendono accettare il rafforzamento della Prussia, e la lotta di liberazione contro Napoleone, culminata nella vittoria di Lipsia del 1813. Nel primo caso è Thomas Mann ${ }^{4}$, nel 1914, a comporre una biografia di Federico, per mettere in luce come allora si sia vissuta una situazione simile a quella del suo tempo e per sottolineare come l'inizio delle ostilità non si configuri affatto come un'aggressione, quando abbia uno scopo difensivo, per prevenire un attacco sicuramente programmato, o perché si risponda a una provocazione palese. Nel secondo caso ci si richiamerà a Fichte, soprattutto per la sua oratoria politica, per i Discorsi alla nazione tedesca, come al filosofo delle guerre di liberazione, che già era stato utilizzato quando si trattava di costruire l'unità nazionale e di infiammare il patriottismo dei tedeschi.

E proprio a Fichte Husserl dedicherà tre lezioni ${ }^{5}$, ripetute tra il novembre 1917 e il novembre 1918, riservate ai partecipanti alla guerra iscritti all'Università di Friburgo e per questo contributo significativo riceverà nel 1918 la croce prussiana al merito per aiuti bellici. In questo momento Husserl condivide il sentire comune e si rivolge a Fichte non tanto per le sue riflessioni

\footnotetext{
${ }^{3}$ Su questi temi si leggano le pagine di MEINECKE, F. Preußen und Deutschland im 19. und 20. Jahrhundert (1918). In: Brandenburg, Preußen, Deutschland. Kleine Schriften zur Geschichte und Politik, Werke. Herausgegeben und eingeleitet von E. Kessel, Bd. 9, Stuttgart: Koehler, 1979; MANN, Th. Pensieri di guerra. In: Scritti storici e politici, Milano: Mondadori, 1957.

${ }^{4}$ MANN, Th. Federico e la grande coalizione. Roma: Treves, 2006.

${ }^{5}$ HUSSERL, E. Fichtes Menschheitsideal. In: Aufsätze und Vorträge 1911-21, a cura di Th. Nenon e H. R. Sepp, Husserliana, Bd. XXV, The Hague, M. Nijhoff, 1986, p. 267-293; tr. it. di F. Rocci, Fichte e l'ideale di umanità: tre lezioni. Pisa: Edizioni ETS, 2006.
} 
teoretiche, che lo rendono uno dei padri dell'idealismo, ma piuttosto per le sue opere: Destinazione dell'uomo e Iniziazione alla vita beata, mentre non si sofferma sui Discorsi alla nazione tedesca, certamente meglio fruibili sul piano della propaganda politica, ma meno adatti a una riflessione filosofica, alla quale Husserl intende pur sempre attenersi. Anzi, leggendo il testo, si ha quasi l'impressione che il generale entusiasmo patriottico contagi il filosofo solo all'inizio e alla fine, mentre, nel corso delle lezioni, l'interesse è tutto rivolto, prima, alla prospettiva teleologica dell'io assoluto e, poi, all'ascesa a Dio, che si autorivela e comunica nei vari livelli di umanità. L'interesse per l'idealismo fichtiano è motivato dal fatto che esso ha determinato un tipo completamente nuovo di interpretazione del mondo, attraverso il quale si pone in contrasto con la concezione tradizionale, propria della scienza positiva e del modo naturale ingenuo di pensare. Grazie a esso il soggetto, che prima era stato solo oggetto passivo delle affezioni, diventa attivo produttore delle oggettività, sicché, eliminata la kantiana cosa in sé, è assolutamente nient'altro che essere agente: «[...] dunque non coincidono soltanto essere soggetto ed essere agente, ma anche essere oggetto per un soggetto ed essere prodotto da un'azione» ${ }^{6}$.

Eppure l'idealismo è apparso agli stessi tedeschi una filosofia del tutto incomprensibile, pur essendo sbocciato in terra tedesca e risalendo solo a un secolo avanti, non a millenni precedenti: la radice di tale misconoscimento è dovuto al dominio esercitato sugli spiriti dalle nuove scienze esatte e dalla cultura tecnica da esse generata. Ora il merito della riscoperta sembra da doversi attribuire proprio alla guerra in corso:

[...] e poi ora è venuta questa guerra, questo destino per la nostra nazione tedesca, alto e gravoso al di là di ogni comprensione. Che evento eccezionale! Viene fondata la prima organizzazione di popoli, estesa quasi a tutta la terra, ma a quale scopo? Per nessun altro, se non per annientare la forza della Germania, per privare il popolo tedesco di una vita, un agire e un operare produttivi. In tutta la storia è mai stato inflitto a un popolo destino più alto, e insieme una prova più ardua? ${ }^{7}$

Una tale epoca deve costituire un'occasione di recupero interiore e di svolta, e deve operare un rinnovamento delle fonti cui attingere energie ideali, ricorrendo alle radici che già in passato hanno offerto alimento e sostegno: «[...] già un secolo fa il nostro popolo tedesco combatté una battaglia per l'esistenza. La

${ }^{6}$ HUSSERL, E. Fichte e l'ideale di umanità, p. 60.

${ }^{7}$ HUSSERL, E. Fichte e l'ideale di umanità, p. 49. 
Germania umiliata a Jena con la Prussia insorse e vinse. Vinse grazie a nient'altro se non alla forza dello spirito nuovo che l'idealismo tedesco e colui che un tempo ne fu il portabandiera, Fichte, vi avevano risvegliato» ${ }^{8}$. Allora furono proprio le idee religiose ed etiche che risvegliarono le energie migliori e trasformarono uomini deboli e meschini in eroi: di fronte a questi ideali perde vigore il modo di pensare positivistico e naturalistico, così come il pericolo e la morte non possono più essere ignorati o mistificati, ma tornano a reclamare i loro diritti. In questa valorizzazione Husserl non ignora, e anzi sottolinea con insistenza, come molti pretenziosi artifici della dottrina della scienza fichtiana siano insopportabili per chi è stato educato a rispettare le esigenze teoretiche di una scienza rigorosa, e osserva che è meglio soffermarsi solo sul famoso oratore patriottico, sul filosofo che ha indagato la morale e Dio.

Un primo guadagno importante sta nel recupero idealista dell'interpretazione platonica del mondo, grazie alla quale la spiegazione possibile della realtà sensibile non è solo quella offerta dai processi della causalità materiale e meccanica, ma rinvia a una necessità teleologica, cioè viene compresa guardando al suo fine ultimo, che, al pari dell'idea platonica del bene, è la fonte di tutti i valori autentici. Rispetto all'uomo dei sensi, che utilitaristicamente ripone la sua felicità nel conseguire il massimo godimento per il maggior numero di persone, il solo valore puro e assoluto risiede nell'azione conforme al dovere e l'uomo, in quanto soggetto di un agire morale, è soggetto libero in una società destinata alla libertà. Sicché il mondo esiste - e il suo senso viene appreso - allo scopo di procurare all'uomo le condizioni di un agire morale; e l'uomo si realizza, conseguendo la sua destinazione e la felicità, in quanto si sa parte di un ordinamento morale e soprasensibile del mondo. Ma Fichte, quasi ripercorrendo il passaggio da Platone al Neoplatonismo, nella seconda fase della sua riflessione, abbraccia una prospettiva religiosa e mistica, che integra la precedente visione, esclusivamente morale; attraverso cinque tappe, procedendo dalla sensibilità e passando per l'eticità, la moralità superiore e la fede, giunge alla contemplazione di Dio, tipica della scienza. L'uomo guadagna la dignità che gli compete, in quanto Dio si rivela nella forma dell'io puro, cioè attraverso una serie infinita di autoriflessioni, con cui si frange nella molteplicità dei soggetti finiti, rappresentandosi prima in forme velate, per poi guadagnare in trasparenza e giungere all'autocoscienza assoluta. Gli uomini, essendo liberi, possono perciò elevarsi e decidersi per i livelli superiori, cioè per Dio; e, d'altro lato, è Dio stesso che in ogni

${ }^{8}$ HUSSERL, E. Fichte e l'ideale di umanità, p. 49-50. 
uomo opera, nell'impulso al soddisfacimento pieno e completo. A Husserl preme di sottolineare come in questa auto rivelazione di Dio nell'uomo, in corrispondenza con i differenti livelli di umanità, si perviene finalmente alla beatitudine, capace di garantire all'uomo il possesso intimo e ultimo dell'oggetto del proprio desiderio. Se, nella sua essenza, ogni vita è beata e se l'unità con l'oggetto del proprio desiderio è amore, allora l'amore è sintomo e sigillo di ogni autentico e pieno soddisfacimento. Inoltre - e su questo punto Husserl insiste particolarmente - ciascuno di noi, partecipando all'essere divino, è una rivelazione individuale di Dio, sicché ciascuno può ascoltare la voce divina e comprendere la sua alta destinazione, dando, con amore, forma all'idea di Dio. La scienza assoluta e compiuta può comprendere pienamente la congiunzione della vita divina e di quella umana, giungendo a una conoscenza onnicomprensiva di Dio, che includerebbe una conoscenza onnicomprensiva del mondo, e del mondo in Dio?.

A questo punto Fichte elabora gli specifici ideali di un uomo, per vedere quale realizzazione pratica possano trovare: in tal modo, negli anni della profonda umiliazione della Germania, egli ha offerto al popolo tedesco un motivo per combattere e sperare, indicando una destinazione personale, che è pure collettiva e nazionale, sicché la liberazione dei singoli e di un popolo coinciderà con la liberazione dell'intera umanità. Per questo motivo Husserl può dire che «[...] il Fichte delle guerre di liberazione parla anche a noi ${ }^{10}$, giacché un popolo, che ha generato tali spiriti, che ha cercato Dio e ha incarnato gli ideali contemplati,

[...] deve essere e rimanere la speranza dell'umanità. Ma che questo si compia veramente nella vita è il compito infinito di tutti noi, di tutti noi che vogliamo vincere in questa guerra perché la rivelazione delle idee divine proceda nel nostro splendido popolo tedesco, in modo che esso cresca sino a raggiungere il vero splendore, che si innalzi in sé e innalzi con se stesso l'umanità intera ${ }^{11}$.

Appare significativo come, anche all'interno di un contesto di accentuata enfasi patriottica e nazionalista, Husserl sottolinei due punti, che poi ritorneranno nella sua riflessione successiva, modulata su toni ben diversi: l'infinità del compito cui si è chiamati, che, quindi, non può essere

\footnotetext{
${ }^{9}$ HUSSERL, Fichte e l'ideale di umanità, p. 87.

${ }^{10}$ HUSSERL, Fichte e l'ideale di umanità, p. 88.

${ }^{11}$ HUSSERL, Fichte e l'ideale di umanità, p. 88-89.
} 
assolto né da una singola generazione, né attraverso un singolo evento; inoltre, l'innalzamento auspicato per il popolo tedesco, dovrebbe comportare un'analoga elevazione per tutta l'umanità.

In questi stessi anni, a partire dal 1915, il tema della guerra, nel rapporto tra la Germania e gli stati europei, viene affrontato con maggiore ampiezza e concentrazione da Max Scheler, un fenomenologo che, a differenza di Husserl, è maggiormente interessato non solo a questioni socio-politiche, ma anche a un coinvolgimento più diretto e personale. In un volume dal titolo emblematico, Il genio della guerra e la guerra tedesca ${ }^{12}$, dedica lunghe riflessioni alle possibili valenze positive della guerra e alle condizioni che, in determinati casi, la rendono necessaria, proprio come quella che è in corso, anche se essa si rivelerà in realtà un evento assai difforme rispetto all'essenza della guerra in precedenza esplicitata. La prima guerra mondiale assume un significato profondo ed epocale, filosofico e metafisico, estremamente arduo e drammatico: chi non avesse, come Scheler stesso, almeno in quegli anni, una fede viva in una potenza sovrumana che tutto regge con saggezza e giustizia, «dovrebbe vedere aprirsi sotto i suoi occhi, in questa radicale esplosione di tutti i legami della cultura e della morale più alte un abisso mai immaginato e un caos mai visto» ${ }^{13}$. Si tratta di vedere se l'Europa sia corrotta nelle sue più profonde radici spirituali, sicché la guerra appare come un'ultima occasione per rinascere dalla colpevole e dilagante infezione capitalistica. L'unità culturale europea aveva la capacità di preservare le molteplici specificità nazionali e l'equilibrio tra le diverse creazioni spirituali, grazie al legame di un comune interesse garantito da tre forze spirituali: gli antichi, il cristianesimo e le stratificazioni culturali delle moderne nazioni europee. Secondo Scheler, la tensione tra le diverse nazioni e l'unità sovranazionale dell'Europa poteva essere attenuata attraverso l'esercizio della solidarietà, intesa come l'assunzione di una responsabilità delle persone, singole e collettive, per le altre persone, al cospetto della persona di Dio. Lo sviluppo storico intrapreso con la modernità, sia pure con modalità e incidenza differenti, ha lentamente corroso i caratteri e le condizioni della vita comunitaria, ispirata ai principi del Cristianesimo, soprattutto a causa del crescente influsso del capitalismo e di una mentalità utilitaristica. Secondo Scheler, il capitalismo non consiste solo in un certo sistema economico, ma,

12 SCHELER, M. Der Genius des Krieges und der deutsche Krieg (1915). in: Politisch-pädagogische Schriften, Gesammelte Werke (GW), Bd. 4, Francke Verlag, Bern 1982, p. 7-250. Si vedano pure i seguenti saggi: Europa und der Krieg (1915), GW, 4, p. 251-266; Der Krieg als Gesamterlebnis (1916), GW, 4, p. 266-282; Die Ursachen des Deutschenhasses (1917), GW, 4, p. 283-372.

${ }^{13}$ SCHELER, M. Der Genius des Krieges..., GW, 4, p. 196-197. 
soprattutto, in un ethos che subordina ai valori dell'utile e del guadagno tutti gli altri valori, privilegia un egualitarismo livellatore ed è animato da una forma di risentimento verso le unità di valore superiore. Un tale cambiamento ha progressivamente dissolto i legami interpersonali e sociali, costitutivi della società europea, sicché a questo punto la guerra, oltre che necessaria, sembra avere anche una funzione medicinale e creativa, poiché spingerebbe gli uomini a ritrovare forme di unificazione; in effetti, contrariamente ai timori nutriti, nel corso della guerra non si è scatenata una lotta, né è esplosa ostilità tra le componenti della società, ma si sono superate diffidenze e contrasti e si è generata una spinta a rafforzare e ad apprezzare la vita comunitaria.

Secondo Scheler, la guerra non deriva dallo scatenamento delle forze belluine e animali sottratte al controllo della razionalità, ma, per essenza, è connessa a un ordine spirituale, superiore a quello meramente animale, sicché in essa non ne va primariamente della vita e della sopravvivenza, ma si tratta di «una lotta per qualcosa di più alto dell'esistenza, una lotta per il potere e per la "libertà" politica, che con quello sta e cade» ${ }^{14}$ e, per questo motivo, a entrare in guerra sono tribù, popoli, stati, nazioni e in palio c'è la perdita di potere da parte di individui e gruppi, il declassamento, la perdita dell'autonomia politica. Presso gli animali c'è la caccia, non la guerra. Pertanto, la guerra non è mera espressione di violenza fisica, che si sostituirebbe al confronto e al colloquio spirituale e razionale; «[...] essa è conflitto di potenza e di volontà tra personalità collettive spirituali, che chiamiamo stati. Questo conflitto di potenza si esprime solo in azioni di violenza, allo scopo di stabilire quali volontà di potere sono degne di dominare» ${ }^{15}$. A questo proposito, Scheler distingue tra potere e violenza e afferma che tra loro sono inversamente proporzionali, sicché di quanto cresce il potere, di tanto diminuisce il bisogno di imporre la propria volontà con la violenza. Quanti tendono a sottovalutare l'essenziale componente spirituale della guerra, in effetti, sopravvalutano la dimensione dell'utile e sono espressione di quella visione utilitaristica tipica del capitalismo, che, a sua volta, rischia di fraintendere anche il fenomeno biologico della vita. Questa non si caratterizza tanto per la sua plasticità e la capacità di adattarsi alle diverse situazioni, quanto, piuttosto, per l'attitudine a muovere, a plasmare e a dominare.

La guerra, pertanto, è chiamata a decidere, secondo la somma giustizia di un diritto divino, quale modello di stato abbia più valore; si

\footnotetext{
${ }^{14}$ SCHELER, M. Der Genius des Krieges..., GW, 4, p. 16.

${ }^{15}$ SCHELER, M. Der Genius des Krieges..., GW, 4, p. 14.
} 
tratterebbe di una specie di diritto divino, in quanto non sarebbero rilevanti le determinazioni più o meno convenzionali dei singoli stati, ma lì troverebbe espressione l'ordine essenziale, logico e oggettivo, di una pura infinita volontà della ragione stessa ${ }^{16}$. Ma chi è il genio della guerra? Non sarà Dio stesso, giacché questi non è un essere umano e, poi, non è una persona collettiva; sarà, piuttosto, la nazione come persona spirituale collettiva o lo stato come espressione istituzionale della corrispondente volontà spirituale collettiva e, in particolare, della volontà di dominio sulla comunità naturale di vita di un popolo ${ }^{17}$. La nazione e lo stato raccolgono le singole persone individue, essendo persone collettive, e ne rendono possibile la realizzazione relazionale e comunitaria, che per quegli individui è costitutiva; in tal modo, anche tali unità complesse sono portatrici di valore e la guerra sarebbe una tendenza intrinseca, non sostituibile con pacifiche forme di contratto, in quanto la società si fonda a partire da una comunità: questa, avendo un'essenza chiusa $\mathrm{e}$ statica, rende difficile l'incontro e la composizione dei contrasti con le forme comunitarie estranee. Mentre la società, istituzionale e stabile, avrebbe una tendenza intrinseca alla composizione e alla pacificazione, l'ethos guerriero della comunità determina una perenne commistione e un ritmico alternarsi di guerra e di pace. La guerra è l'espressione dello spirito di una comunità, a seconda dello scopo che conferisce alla forza direzione e obiettivi e rispetto al quale si separano e contrappongono i contendenti; essa serve all'attuazione di valori sostenuti da una comunità umana e diventa necessaria quando valori culturali, come diritto, filosofia e arte, sono sottomessi a valori della civilizzazione, come tecnica, utilità. Lo stesso valore della sopravvivenza non può essere difeso al prezzo di sacrificare i valori spirituali superiori e chi accetta di mettere a repentaglio la propria vita, e ne paga il prezzo, unisce al diritto anche il potere e la forza, e al conflitto che ne consegue - una guerra giusta - può essere dato il nome di "guerra tedesca". La guerra porta così a una purificazione e a un conferimento di senso su valori reali, mostrando la crisi interna di cui soffre un certo sistema di valori, che va ricondotto alle sue fonti interiori e rivitalizzato; consente una difesa della libertà di seguire decisioni di valore spirituale e culturale, che animano la propria comunità; diventa così il veicolo dello spirito verso la massima signoria e si qualifica come un fenomeno tipicamente umano, costitutivo dell'essenza stessa degli uomini, nelle forme di

\footnotetext{
${ }^{16}$ SCHELER, M. Der Genius des Krieges..., GW, 4, p. 86.

${ }^{17}$ SCHELER, M. Der Formalismus in der Ethik und die materiale Wertethik, GW, Bd. 2. Bern: Francke, 1966, p. 533; tr. it. di G. Caronello, Il formalismo nell'etica e l'etica materiale dei valori. Cinisello Balsamo: Edizioni San Paolo, 1996, p. 664.
} 
vita sociale e collettiva. E proprio lo stato è portatore della persona spirituale collettiva.

Ma le grandi imprese possono chiedere prezzi assai alti, anche in termini di perdite, di dolori e di morti: si ha sacrificio, quando la realizzazione di un bene di valore superiore dipende da o è connessa con la soppressione o la riduzione di un bene dal valore inferiore. Il dolore assume una funzione vicaria, in quanto ogni sofferenza individuale consente che il tutto soffra di meno e per questo motivo lo stato e il popolo possono esigere anche la vita dell'individuo, visto che essi oltrepassano infinitamente la vita del singolo ${ }^{18}$; tuttavia, questo può accadere solo a condizione che la personalità individuale stessa, nel suo nucleo profondo, non sia identica a questa vita, che cioè l'anima sia pensata come immortale.

In tale contesto la guerra diventa allora l'ultimatum divino all'Europa, l'ultimo richiamo del destino, l'ultima occasione offerta affinché essa prenda coscienza dell'anarchia della sua esistenza, si accorga di aver smarrito l'ideale di un'autentica comunità spirituale europea e ritorni alle più intime radici della sua essenza ${ }^{19}$. Questa non è ispirata a forme di internazionalismo ideologico di stampo capitalista o socialista, incapaci di generare una comunità di vita e di amore, ma rinvia a un cosmopolitismo in cui una persona guarda all'altro, lo comprende e gli tende la mano, in quanto entrambi hanno lo stesso obiettivo e tendono alla stessa meta. Si apre la possibilità di un nuovo e positivo europeismo, capace di custodire le identità nazionali, senza decadere né nei nazionalismi chiusi, né in un internazionalismo meramente formale. «Mediante il fatto che ogni nazione pone in atto ciò che le è più proprio portandolo all'espressione più pura e ingenua, si completano le opere, i lavori, le arti, le filosofie, le scienze, le regole delle nazioni in una sempre più compiuta rappresentazione complessiva dello spirito umano donato da Dio ${ }^{20}$. La comunità europea non potrà essere fondata solo su un contratto, ma richiederà una comune base culturale: se tale meta sarà conseguibile solo partendo dalla guerra, e dal suo carico di dolore e morte, ciò attesta la confusione in cui versa lo spirito europeo e, insieme, mette in luce come uno dei risultati di questa guerra potrà anche essere quello di evitare guerre future.

\footnotetext{
${ }^{18}$ SCHELER, M. Der Krieg als Gesamterlebnis..., GW, 4, p. 281.

${ }^{19}$ SCHELER, M. Europa und der Krieg, GW, 4, p. 253.

${ }^{20}$ SCHELER, M. Der allgemeine Begriff von "Nation” und die konkreten Nationalideen (circa 1916). In: Schriften zur Soziologie und Weltanschauungslehre, GW, Bd. 6. Bern: Francke, 1963, p. 346.
} 


\section{DOPO LA FINE DELLA GUERRA: BASI PER LA RINASCITA}

Le modalità in cui l'evento bellico è stato condotto, oltre al suo esito, spingono Scheler a una profonda revisione delle prospettive ora delineate, che in alcuni momenti sembravano toccare, insieme a un'intensa esaltazione retorica, anche punte di freddo cinismo. In alcune pagine dedicate all'idea della pace e al pacifismo, nell'ambito di una riflessione sulla pace perpetua ${ }^{21}$, abbandona la convinzione che la guerra abbia sempre un valore metafisico positivo e affronta invece la questione se non sia la pace a essere un valore positivo per l'individuo e per lo stato; inoltre, se e a quali condizioni essa sia concretamente realizzabile. Contrariamente agli anni precedenti, in cui riteneva che capitalismo ed espansionismo russo fossero i nemici da combattere e che la Germania, in cui le diverse rivoluzioni borghesi non si erano verificate, avrebbe potuto conservare l'articolazione della società secondo gli stati sociali, in una maniera che sembrava più conforme anche alla tradizionale idea cristiana di società, ora Scheler, pur mantenendo costantemente lo sguardo fisso sull'Europa, non ne considera la contrapposizione inconciliabile con altri stati e culture, ma ne rileva il positivo contributo che essa può offrire al concerto delle nazioni mondiali. In questa prospettiva, lo stato nazionale non rappresenta più il caso paradigmatico di una persona collettiva spirituale e la guerra si rivela un mero evento politico, senza la capacità di garantire una purificazione, di legittimare i valori o di indicare una differenza di grado tra $i$ valori. Inoltre, si fa meno stretto quel nesso tra vita umana, potere e guerra, tra forza e diritto, che prima aveva determinato il significato metafisico positivo della guerra, né la storia umana si può ricondurre semplicisticamente a un processo evolutivo dominato dall'aspirazione al potere. Anzi, quanto più le forme d'essere sono elevate, tanto minore è il peso esercitato dall'istinto di potenza, giacché la lotta tra i gruppi umani si riduce sempre più, a favore di una cooperazione collettiva nella comune lotta contro gli enti infraumani. La dimensione bellica non è più collocata nell'essenza della natura umana e la lotta propria degli uomini non è il violento conflitto di interessi, tipico di quelle comunità che difendono solo valori inferiori, come quelli dell'utile o i valori vitali. E nel momento in cui, in tale contesto, lo stato assolutizza la sua sovranità, diventando signore di tutte le comunità, perde il ruolo guida di natura spirituale, per essere solo espressione di forze economiche, e non ha

${ }^{21}$ SCHELER, M. Die Idee des Friedens und der Pazifismus (1931). Bern: Francke, 1974; pure in: Schriften aus dem Nachlaß, Bd. 4, Philosophie und Geschichte, GW, 13. Bonn: Bouvier, 1990, p. 77-121. 
il diritto di chiedere il sacrificio di una vita individuale per la conservazione della totalità.

Quali prospettive di riscatto si dischiudono allora alla ricostruzione spirituale e culturale dell'Europa? Se la guerra non è più il mezzo privilegiato, ancorché doloroso, per pronunciare un giudizio valoriale, quali percorsi andranno seguiti? Su questi punti la revisione compiuta da Scheler è profonda, sia negli anni in cui si sente vicino alla visione cristiana, sia quando progressivamente se ne allontana in maniera sempre più netta, nella fase finale della sua riflessione. Mentre la guerra volge al termine e la Germania deve affrontare una situazione drammatica e incerta, Scheler riflette su due temi, che possono apparire reciprocamente lontani, ma, dal suo punto di vista, sono strettamente connessi: l'idea cristiana dell'amore nel mondo contemporaneo e la ricostruzione culturale dell'Europa, collocata sullo sfondo di una rinascita resa possibile dal pentimento ${ }^{22}$. I toni enfatici e corruschi, presenti negli scritti di soli due anni prima, sono assai lontani, sostituiti da una consapevolezza addolorata e sbigottita, ma sempre appassionata e pienamente coinvolta e convinta nelle proposte avanzate; in tutto questo cambiamento di posizione, assai netto, stupisce che la stessa sicurezza di intuire e afferrare i momenti essenziali del fenomeno studiato suffragasse le analisi di allora e accompagni anche quelle di oggi, di segno del tutto diverso, così come meraviglia che il filosofo non si senta in dovere di fare i conti, sul piano teorico delle argomentazioni addotte, con le tesi sostenute solo poco tempo prima, non tanto allo scopo di cercare od offrire una giustificazione morale, quanto piuttosto per meglio chiarire e spiegare le proprie attuali posizioni. In questo caso, infatti, non si tratta di testimoniare un radicale cambiamento di mentalità, un'avvenuta e vissuta conversione, ma, sul piano filosofico, occorre soprattutto offrire argomenti che sostengano una conclusione e mettano in rilievo le carenze presenti nella tesi opposta, sostenuta in precedenza.

Quale è il presupposto morale per una ricostruzione culturale dell'Europa, e non solo della Germania, dopo una guerra così disastrosa? Lo sguardo si amplia e quelle contrapposizioni prima considerate essenziali, che da sole avrebbero giustificato la guerra, ora lasciano spazio a una considerazione comune, che non vuole alimentare ulteriori divisioni, andando alla ricerca del

${ }^{22}$ SCHELER, M. Die christliche Liebesidee und die gegenwärtige Welt (1917). In: Das Ewige im Menschen, GW, 5, Bonn: Bouvier, 2000, p. 357-401; tr. it. di P. Premoli De Marchi: L'eterno nell'uomo. Milano: Bompiani, 2009, p. 881-981; Id., Vom kulturellen Wiederaufbau Europas, (1917), GW, 5, p. 405-447; tr. it., Ibi, p. 985-1081; Id., Reue und Wiedergeburt (1917), GW, 5, p. 29-59; tr. it., Ibid., p. 153-221. 
colpevole, del presunto unico colpevole (in questo modo si riaprirebbero le antiche contese e le vecchie ferite), ma tenta la strada di una conciliazione, nel condiviso pentimento per la consapevolezza che tutti si sta sotto la colpa e se ne è responsabili.

E affermo che una ricostruzione culturale è possibile solo se, all'interno dei popoli europei, una parte sempre più grande impara a considerare tutto questo avvenimento come una conseguenza di una colpa comune dei popoli europei basata sulla reciprocità - come una colpa, dunque, che può essere abolita e superata interiormente solo attraverso una penitenza comune, un pentimento comune, un sacrificio comune, eliminata solo in virtù di un'opera di ricostruzione, un aiuto, un agire cooperativo che si integrino reciprocamente, e nello spirito di una responsabilità solidale, e che può essere sostituita dai beni di una nuova positiva comunità culturale. Queste tre cose -colpa comune, pentimento comune e volontà comune di ricostruzione - sono strettamente interdipendenti e non devono essere separate sulla base del principio riconosciuto da tutti, secondo il quale ogni stato, ogni popolo, ogni nazione hanno una "posizione" specifica ad essi assegnata nel giardino di Dio e sono chiamati ad offrire un contributo insostituibile e non sostenibile da altri ad una cultura mondiale, e secondo il quale l'Europa, in questa splendida cooperazione dell'umanità storica, rappresenta un'area culturale particolare, relativamente unitaria, con doti e compiti specifici ${ }^{23}$.

Sarebbe gravemente sbagliato chiedersi quale popolo sia stato il responsabile della guerra e il colpevole della sua esplosione, quasi che fosse scontata la possibilità che sussista un solo colpevole, facilmente individuabile da parte di tutti, a partire dal presunto reo. La domanda corretta, invece, non riguarda l'evento reale e il suo inizio effettivo, ma come sia stato possibile che un tale fatto sia avvenuto proprio in Europa, in quel continente con la sua storia e le sue tradizioni. Filosoficamente si indaga l'essenza di un fenomeno e le sue condizioni di possibilità, per cercarne il contesto morale, più che gli episodi effettuali, che possono essere raccontati, ma non spiegano. Della guerra la responsabilità è reciproca e comune, e sarebbe un grave atto di fariseismo affermare che solo alcuni popoli hanno voluto e causato la guerra; in effetti, tutti i popoli la volevano, sia pure per differenti motivi e con contrapposti obiettivi, e nessuno parteggiava sinceramente, e non solo verbalmente, per la pace. Né sarebbero responsabili solo i governi, che avrebbero costretto i loro

${ }^{23}$ SCHELER, M. Vom kulturellen Wiederaufbau Europas, p. 416; tr. it., p. 1010-1011. 
popoli riluttanti a combattere; invece, le popolazioni hanno partecipato in modo convinto, sostenendo e incoraggiando i loro governanti $\mathrm{i}^{24}$.

Il pentimento comune consiste in una volontà di pentimento e di sacrificio: soprattutto il pentimento è, per Scheler, un atteggiamento fondamentale allo scopo di penetrare in profondità nel passato di un essere umano; è una forza «[...] che scopre la nostra essenza intima, liberandola e illuminandola, nella nostra storia. Anzi, essa sola rende possibile una conoscenza storica che non si limiti a descrivere il passato, ma che faccia la cosa più importante che la conoscenza storica possa fare - liberare dal passato e rendere la nostra anima libera e forte per un futuro e un'attività nuovi $\aleph^{25}$. Il pentimento, infatti, esige un raccoglimento in noi stessi e nel nostro passato, e rende capaci di rivedere la propria esistenza nel suo complesso, nel volto che ha assunto, nelle scelte e negli orientamenti fondamentali, di comprenderne il senso e di trascenderla, liberandosene, per mutare, anche radicalmente, direzione. Certamente il passato rimane immodificabile e nulla può far sì che esso non sia avvenuto, né che i suoi effetti non si siano dispiegati; tuttavia, la nostra esistenza personale non è una sorta di fiume che scorre secondo un'unica direzione, quella dei fenomeni naturali, giacché, in questo caso, nessun ripiegamento sul passato e nessuna ricomprensione globale di esso sarebbero possibili.

Nell'esperienza di ciascun momento della nostra vita temporale indivisibile sono co-presenti la struttura e l'idea della totalità della nostra vita e della nostra persona. Ciascuno di questi singoli momenti della vita, che corrisponde ad un unico punto indivisibile del tempo oggettivo, ha in sé le sue tre dimensioni del presente vissuto, del passato vissuto e del futuro, il cui dato si costituisce nella percezione, nel ricordo immediato e nell'attesa immediata. Grazie a questo fatto meraviglioso non è la realtà, bensì piuttosto il senso e il valore della totalità della nostra vita, nella sfera del nostro libero potere in ciascun momento della nostra vita ${ }^{26}$.

In tal modo, ciascuno crea con il proprio passato una relazione che non è di estraneità e neppure di deterministica dipendenza; se la realtà del passato non può essere mutata o annullata, il suo senso, all'interno della propria vita e della propria storia, può essere ripreso e rigenerato, in quanto è

\footnotetext{
${ }^{24} \mathrm{Su}$ questo punto si vedano le acute e documentate osservazioni di CANFORA, L. 1914. Palermo: Sellerio, 2014, soprattutto le p. 78-88.

${ }^{25}$ SCHELER, M. Vom kulturellen Wiederaufbau Europas, p. 417; tr. it., p. 1011-1013.

${ }^{26}$ SCHELER, M. Reue und Wiedergeburt, p. 33; tr. it., p. 163.
} 
sottoposto alla forza della persona: ne deriva la possibilità di un rapporto con il proprio passato che non è di dipendenza risentita, ma di assunzione capace di cambiamento.

Infine, la ricostruzione non va intesa come il semplice ristabilimento dei rapporti sussistenti prima della guerra, giacché non esistevano allora veri legami spirituali, come è provato dal fatto che si sono dissolti e, in questo caso, la guerra non è stata la causa della loro rottura, ma ha consentito che la malattia, loro intrinseca, esplodesse e fosse diagnosticata. Almeno per questo aspetto, la guerra ha svolto quindi una funzione positiva! Nella guerra non si è constatato il fallimento della concezione cristiana dell'amore, ma si è visto che da tempo ormai tale concezione non innervava più intimamente la cultura europea, in quanto era stato confuso con una versione dell'umanitarismo e aveva perduto l'intrinseca sua essenza comunitaria. Quando sussiste vera amicizia, essa permane anche nella lotta tra interessi opposti degli amici; in Europa, prima della guerra, c'era solo cortesia diplomatica, una buona dose di ipocrisia, la condivisione di interessi comuni, una reciproca adulazione e una condivisa illusione sullo stato di salute complessivo del continente. Per questi motivi, si rende necessario ora un deciso cambio di mentalità, senza il quale sarebbe alto il rischio che si riproducano, accresciuti, i mali del recente passato e che non riesca a consolidarsi una comunità culturale propriamente europea. Questa non è incompatibile con un sano e vero cosmopolitismo, giacché esso è reso possibile dalla convinzione che tutte le individualità nazionali $\mathrm{e}$ popolari sono insostituibili e indispensabili nella costruzione di una cultura umana comune e integrata. Cultura nazionale e cosmopolitismo sono i due lati di una stessa verità e si oppongono alle pretese, purtroppo ancora vive, anche se momentaneamente assopite, di dominare il mondo da parte di una sola nazione o di un singolo stato. Un tale mutamento consentirà anche di ritrovare la forza dell'unità dell'Europa e delle sue culture, e di capire come possano essere messe in luce e rafforzate.

A una tale ricostruzione dovrebbero collaborare tutte le forze spirituali europee, che nel corso della storia sono state attive e incisive. In particolare, tre ne vengono ricordate da Scheler: la cultura antica, il cristianesimo; specie nella sua versione agostiniana, che guarda all'amore e alla realizzazione del regno di Dio nel mondo, più che alla contemplazione e alla fuga dal mondo; l'età moderna, a partire dal Rinascimento, con il suo intreccio di arti, lettere, scienze e tecniche. Va pertanto perseguita la connessione e la reciproca influenza tra le culture nazionali europee, ma va anche accuratamente evitata un'educazione 
su base essenzialmente positivistica o nazionalistica ${ }^{27}$. In questo senso, va evitata la chiusura in se stesso dello spirito germanico, attraverso il culto del mito germanico e un'attenzione unilaterale alla storia della patria. L'antichità non va isolata né dalla preistoria asiatica e neppure dall'ellenismo e dal cristianesimo; mentre va favorito l'inserimento delle persone in una pluralità dio comunità, contemporaneamente, ciascuna con propri compiti e scopi e con la disponibilità di ognuna a sacrificarsi per il giusto sviluppo delle altre. Oltre al riferimento alla classicità della Grecia e di Roma, occorre rivalutare la cultura dell'Europa orientale, allo scopo di completare l'individualismo tedesco con il senso comunitario slavo, l'orientamento alla razionalità con la sensibilità mistica, giacché le forme culturali occidentali e orientali, per sé prese, sono imperfette e necessitano un'integrazione. Inoltre, la filosofia non dovrà seguire la tradizione kantiana, nonostante i suoi meriti; «[...] abbiamo bisogno di una filosofia che assomigli non, come quella kantiana, a un pugno chiuso, ma a una mano aperta; che si riallacci alla grande eredità del mondo ideale europeo-cristiano e la cui anima debba al contempo essere il più rigoroso oggettivismo e il riconoscimento dei fatti e delle connessioni essenziali ultime nel mondo e nello spirito umano» ${ }^{28}$.

Scheler assegna, poi, un ruolo fondamentale nella ricostruzione europea al cristianesimo, colto nella polarità delle sue versioni occidentale $\mathrm{e}$ orientale, ma dovrà essere chiaramente affermata la separazione della chiesa dallo stato, con tutte le conseguenze, che, se al momento potranno apparire scomode e spiacevoli, favoriranno, poi, una purificazione verso un autentico spirito religioso. Parimenti, criticando gli eccessi del capitalismo, la Chiesa dovrà favorire lo sviluppo di un cristianesimo sociale, per il quale, in quegli anni, sembrano ridursi le consuete difficoltà, anche perché i molti idoli terreni, che le diverse ideologie proponevano come facilmente realizzabili a vantaggio dell'umanità, sono stati messi alla berlina dalla furia devastatrice della guerra.

Se nell'immediato primo dopoguerra Scheler vede nella religione cristiana una forza aggregatrice assai potente e auspica una sua collaborazione con i movimenti socialisti, all'insegna della solidarietà, successivamente, allontanatosi dalla Chiesa e dall'esperienza di fede, sottolinea maggiormente il "livellamento" (Ausgleich) tra le culture, come tipico del momento contemporaneo, e all'Europa non competerà più una posizione pionieristica, capace di resistere al movimento mondiale verso la civilizzazione, La

\footnotetext{
${ }^{27}$ SCHELER, M. Vom kulturellen Wiederaufbau Europas, p. 423; tr. it., p. 1025.

${ }^{28}$ SCHELER, M. Vom kulturellen Wiederaufbau Europas, p. 434; tr. it., p. 1051.
} 
coscienza europea non è più un'evidenza assoluta e indubitabile, colta tramite l'intuizione fenomenologica, ma un impegno costruttivo di lunga lena, alla cui attuazione sono necessarie nuove istituzioni e molto lavoro. La nazione non appare più come una persona spirituale collettiva, di valore fondamentale, ma può diventare un pregiudizio che ostacola lo sviluppo dello spirito. Il livellamento progressivo interesserà, con l'Europa, i grandi paesi dell'Asia, e la cultura europea dovrà essere osservata a partire dalle altre culture, in modo che essa possa contribuire alla solidarietà reciproca tra tutti gli esseri viventi e di tutti gli spiriti nello spirito eterno, la cui realizzazione costituirà l'obiettivo stesso della storia.

\section{RiNnOVAMENTO, METODO E TELOS DELl'UMANITÀ}

Anche Husserl, che nelle lezioni su Fichte aveva mostrato di condividere il generale sentimento favorevole alla guerra, intesa come occasione di rinascita e di difesa della cultura autentica, sia pure con accenti diversi da quelli di Scheler e tenendosi prevalentemente a una trattazione teoretica del tema, già nel corso dei combattimenti e, poi, alla fine, dopo la sconfitta e il trattato di pace, soprattutto nell'epistolario, rivede nettamente le sue posizioni. In precedenza, aveva esaltato il sentimento di unità che, anche a rischio di sacrificare la propria vita, legava fra loro i soldati sul fronte di battaglia, rendendo tangibile come in quei momenti non si vivesse più da persone private, ma la sofferenza individuale venisse sublimata e innalzata a un livello sopra-individuale. Ora, invece, afferma che la guerra non ha costituito un momento di rinascita, ma ha reso esplicita la decadenza e la miseria morale e filosofica in cui si è precipitati, perdendo ogni eventuale significato etico; in essa l'umanità si è macchiata di una colpa universale e profonda ed è dilagato un odio crescente tra i popoli europei; le modalità e i contenuti, secondo cui la pace è stata imposta, hanno poi ulteriormente aggravato la situazione, ponendo le premesse per negativi sviluppi nel futuro. In una serie di articoli, scritti negli anni 1922-23 e poi pubblicati sulla rivista giapponese Kaizo nel 1923-24 $4^{29}$, Husserl si mostra consapevole di quella crisi, su cui in quegli anni riflettono molti pensatori e intellettuali europei, e invoca un rinnovamento soprattutto filosofico, di cui delinea anche le condizioni di possibilità. Più

${ }^{29}$ Gli articoli, compresi quelli che non apparvero sulla rivista, sono oggi pubblicati in: HUSSERL, E. Aufsätze und Vorträge (1922-1937), Husserliana, a cura di Th. Nenon e H.R. Sepp, Bd. 27. Dordrecht: Kluwer, 1989, p. 3-94; tr. it. di C. Sinigaglia, L'idea di Europa. Milano: Cortina, 1999, p. 3-110. L'edizione italiana contiene anche cinque delle aggiunte contenute nell'edizione originale. 
tardi, in un articolo del 1923 e in una conferenza a Vienna del 7 maggio $1935^{30}$, colloca all'interno di un più ampio e radicale cammino di pensiero la possibilità di uscire dalla crisi, che l'umanità europea attraversa, dopo molti anni dal primo dopoguerra, mentre in Germania, conclusasi una fallimentare esperienza repubblicana, è andato al potere il governo totalitario di Hitler.

La prima guerra mondiale aveva sconfitto il mito del progresso, che, inarrestabile, avrebbe dovuto seguire agli sviluppi e alle applicazioni delle scienze, eliminando conflitti e favorendo incremento e diffusione del benessere: molti pensatori, allora, credono di rimediare ai difetti dello scientismo e al predominio della tecnica, esaltando, in contrapposizione allo spirito, la vita, che sarebbe esuberante e contraddittoria e che dalle regole della ragione sarebbe coartata e impedita. Su questa strada non si incammina certo Husserl, fedele alla sua formazione matematica e al culto della ragione, intesa però in maniera non unilaterale.

Egli non pone un contrasto tra la vita e lo spirito, giacché l'umanità autentica è tutta permeata dalla razionalità e ne segue le regole; si tratta di

[...] un'umanità che si concepisce come un'umanità razionale, un'umanità che comprende di essere razionale nel voler-essere-razionale, che comprende che ciò significa l'infinità della vita e degli sforzi verso la ragione, che la ragione sta a indicare proprio ciò verso cui l'uomo, in quanto uomo, tende nel suo intimo, ciò che soltanto lo può pacificare, che può renderlo "felice", che la ragione non può essere distinta in ragione "teoretica", "pratica" ed "estetica" e simili, che l'essere-uomo implica un essere-teleologico e un dover-essere, e che questa teleologia domina ogni azione e ogni progetto egologico ${ }^{31}$.

Da questo passo alcuni elementi emergono con chiarezza, che altrove vengono poi sviluppati e ampiamente giustificati: la razionalità piena e autentica non è un fatto naturale, quasi innato, ma esige un impegno, un voler

${ }^{30}$ HUSSERL, E. Die Idee einer philosophischen Kultur. In: Erste Philosophie, a cura di R. Boehm, Husserliana, Bd. 7, Den Haag: M. Nijhoff, 1956, p. 8-17; 203-207; tr. it. di R. Cristin, Crisi e rinascita della cultura europea. Venezia: Marsilio, 1999, p. 29-44; Id., Die Krisis der europäischen Menschentums und die Philosophie. In: Die Krisis der europäischen Wissenschaften und die transzendentale Phänomenologie, a cura di W. Biemel, Husserliana, Bd. 6, M. Den Haag: Nijhoff, 1954, p. 314-348; tr. it. di E. Filippini, La crisi dell'umanità europea e la filosofia. In: La crisi delle scienze europee e la fenomenologia trascendentale. Milano: Il Saggiatore, 1961, p.328-358; pure in: Crisi e rinascita della cultura europea, p. 47-92, da cui si citerà.

${ }^{31}$ HUSSERL, La crisi delle scienze europee e la fenomenologia trascendentale, p. 289-290. 
essere razionali, che implica un dovere e uno scopo, un telos, raggiungibile, come presto vedremo, attraverso un metodo preciso. Naturale sarà allora la tendenza a tale realizzazione, che però non è immediata o automatica, ma è destinata ad avvenire - e questo è un altro elemento - attraverso un percorso e una storia, personale, ma anche sociale e culturale; il cammino attraverso cui la razionalità si compie, proprio perché comporta impegno personale e metodo, non è lineare o garantito nel suo risultato e può subire rallentamenti e regressi. Infine, tale razionalità non separa la dimensione teoretica da quella pratica ed estetica, né esalta unilateralmente un aspetto, assolutizzandolo nei confronti degli altri e, pertanto, è in grado di pacificare e di rendere felici.

Infatti, «la riforma etica di Socrate è caratterizzata dall'aver inteso come vita veramente appagante una vita fondata sulla pura ragione. Ciò significa: una vita in cui l'uomo esercita, in un'incessante autoriflessione e in una critica - una critica che sviluppa un'analisi ultimativa - dei propri scopi vitali e quindi naturalmente, mediata da essa, una critica delle proprie vie e dei propri mezzi vitali». Un tale processo conoscitivo comporta un ritorno all'evidenza pura e alla comprensione intuitiva come fonti del sapere autentico, il quale, «[...] prodotto originariamente dalla piena evidenza, è l'unico, insegna Socrate, che renda l'uomo pienamente virtuoso; oppure, il che è lo stesso, l'unico che possa procurare all'uomo una felicità effettiva, la massima soddisfazione possibile» ${ }^{32}$. Questa vita personale desta, lucida e consapevole, frutto della continua riflessione su di sé, si contrappone all'opinione oscura e prevenuta ed è frutto di un metodo universale della ragione, capace di conseguire la necessaria chiarificazione attraverso la riconduzione all'evidenza apodittica.

Così si può ottenere quel "rinnovamento", che

[...] è l'appello generale del nostro tormentato presente, e nell'intero ambito della cultura europea. La guerra, che dal 1914 l'ha devastata e che dal 1918 non ha fatto altro che sostituire i mezzi della coercizione militare con quelli più "raffinati" della tortura psicologica e dell'indigenza economica, non meno depravanti dal punto di vista morale, ha rivelato l'intima non verità e insensatezza di tale cultura. Proprio questa rivelazione, però, finisce per impedire che essa dispieghi appieno la sua autentica forza. Una nazione, un'umanità vive e opera nella pienezza delle forze soltanto se sorretta nel suo slancio da una fede in se stessa e nella bellezza e bontà della vita della propria cultura $^{33}$.

\footnotetext{
${ }^{32}$ HUSSERL, Die Idee einer philosophischen Kultur, in Crisi e rinascita della cultura europea, p. 34-35.

${ }^{33}$ HUSSERL, L'idea di Europa, p. 3.
} 
Una tale fede, che è stata anima della cultura tedesca, è andata perduta proprio a causa della guerra, dei suoi esiti e dei gravi mali spirituali di cui essa era sintomo. Quella fede potrà essere ritrovata e potrà smuovere le montagne - ottenere cioè risultati miracolosi - «solo se si trasforma in un pensiero obiettivo, razionalmente evidente, solo se giunge a chiarire e a determinare pienamente l'essenza e la possibilità del suo fine, nonché del metodo che permette di realizzarlo» ${ }^{34}$. A questo punto il richiamo di Husserl è duplice, storico-genetico e metodologico. Dal primo punto di vista, egli ricorda come l'Europa, considerata nella sua forma spirituale, conserva

\begin{abstract}
[...] innata un'entelechia, che permane attraverso tutti i mutamenti delle forme di vita europee e conferisce ad essi il senso di uno sviluppo verso quella forma di vita e di essere che costituisce il suo eterno polo ideale. [...] L'umanità spirituale non è mai compiuta e mai lo sarà, non potrà mai ripetersi. Il telos spirituale dell'umanità europea, nel quale è incluso il telos particolare delle singole nazioni e dei singoli uomini, è in una prospettiva infinita, è un'idea infinita verso cui tende segretamente, per così dire, l'intero divenire spirituale ${ }^{35}$.
\end{abstract}

Si tratta, quindi, di una meta ideale, mai effettivamente raggiunta una volta per tutte, che si sviluppa nella storia e che, una volta pervenuta alla coscienza e divenuta consapevole, costituisce un fine pratico e concreto della volontà e assurge quasi a un nuovo livello dello sviluppo evolutivo dell'uomo. Un tale esito cui la storia tende e in tale processo in cui la storia consiste, hanno un preciso luogo di nascita: l'antica Grecia, allorché in essa si è delineato un nuovo atteggiamento verso il mondo circostante, dal quale derivò una nuova formazione spirituale e culturale che fu chiamata filosofia. Essa volge il suo interesse al tutto, colto nelle sue caratteristiche più universali, al problema del divenire, e tende poi a specificarsi e a ramificarsi in quelle che saranno le singole e differenti scienze; si propone di elaborare teoria, raccogliendo tutte le verità in sé valide in un'unità sistematica connessa, atta a costituire l'unità di un'unica scienza universale.

Un tale atteggiamento teoretico, che ispira il modo di essere e la volontà di singoli, gruppi e comunità e delinea un compito tendenzialmente

\footnotetext{
${ }^{34}$ HUSSERL, L'idea di Europa, p. 5.

${ }^{35}$ HUSSERL, La crisi delle scienze europee e la fenomenologia trascendentale, p. 333-334.
} 
infinito, ha la sua origine storica presso i greci ${ }^{36}$. Esso dà anche origine a un tipo di uomo nuovo, che di fronte al mondo si pone in un modo originale, in quanto solleva domande e cerca risposte che hanno caratteri fino ad allora sconosciuti. Alla cultura europea, nata dalla Grecia, inerisce un costante orientamento secondo una norma, che permea la vita delle singole persone, delle nazioni e delle società particolari e che produce un andamento necessario nello sviluppo dello spirito: se questa precisazione meglio determina quel tipo nuovo di umanità, di cui si diceva, mette pure in luce un momento decisivo e rilevante, su cui Husserl pone l'accento e che costituirà un permanente motivo ispiratore del suo lavoro, al di là dei mutamenti e delle variazioni. Si tratta del ruolo svolto dalle idee e dalla dimensione eidetica, che costituisce uno dei punti di forza dell'analisi fenomenologica:

[...] le idee, queste nuove e meravigliose formazioni di senso prodotte da singole persone, che nascondono in sé infinità intenzionali, non sono come le cose reali nello spazio, le quali, per gli uomini in quanto persone, non significano ancora qualcosa per il semplice fatto di presentarsi nel campo della loro esperienza. Con la prima concezione delle idee, l'uomo diventa a poco a poco un uomo nuovo. Il suo essere spirituale entra nel movimento di una progressiva trasformazione. [...] In esso (e successivamente anche al di là di esso) si diffonde anzitutto una particolare umanità, che, vivendo nella finitezza, vive protesa verso i poli dell'infinità ${ }^{37}$.

A questo punto interviene il secondo momento del richiamo husserliano, quello metodologico: il rinnovamento, richiesto dalle urgenze della storia, esige una trasformazione, non meramente istintiva, ma fondata su una razionalità atta a comprendere i diversi fatti empirici, in quanto a essi si applica, ma da essi non viene ricavata o dedotta. Il richiamo a un'indicazione di metodo diventa per Husserl imprescindibile, in quanto da esso dipende non solo la struttura e la consistenza di una teoria, ma anche un conseguente impegno etico sul piano personale e sociale, e, quindi, la trasformazione e il rinnovamento auspicati.

Per quanto concerne il metodo, può essere realmente fecondo un solo genere di considerazioni, quello che può essere rappresentato come considerazione d'essenza, e soltanto questo può dischiudere la via a una scienza razionale tanto dell'uomo in generale quanto del suo "rinnovamento". Se, però, ci si

\footnotetext{
${ }^{36} \mathrm{Si}$ veda in proposito: HUSSERL, E. Die Idee einer philosophischen Kultur, in Crisi e rinascita della cultura europea, p. 38-39, e La crisi delle scienze europee e la fenomenologia trascendentale, p. 338-339.

${ }^{37}$ HUSSERL, La crisi delle scienze europee e la fenomenologia trascendentale, p. 335.
} 
rende conto che un "rinnovamento" appartiene, in maniera essenzialmente necessaria, allo sviluppo di un uomo e di un'umanità verso la vera humanitas, risulta allora che la fondazione di questa scienza costituirebbe il presupposto necessario per un effettivo rinnovamento, anzi un primo inizio della sua stessa messa in scena. In ogni caso, l'unica cosa che per il momento possiamo fare è prepararlo ${ }^{38}$.

Finora il luogo precipuo di un'indagine di essenza è la matematica; il compito che attende un'umanità in cerca di trasformazione (e non soltanto $\mathrm{i}$ filosofi di professione!) e, allora, quello di applicare tale metodo alle scienze dell'uomo ed elaborare concetti come quello di uomo in generale quale essere razionale, di comunità e suo componente, di famiglia, popolo e stato, ecc. Tali essenze non sarebbero induttivamente fondate, a partire da regolarità empiriche, ma, muovendo da singolarità di oggetti pensati nella fantasia, si cerca quanto permane identico nella libera e arbitraria variazione cui essi sono sottoposti entro l'infinità di ciò che è idealmente possibile. "L'identico che permane in tale variazione emerge nella sua evidenza, quando si percorra con lo sguardo l'infinità aperta delle sue varianti, come l'identico che le attraversa, come la loro "essenza" comune, la loro "idea" ${ }^{39}$. In tal modo, la cultura rigorosa, e in questo senso scientifica (nel senso cioè dell'episteme greca), è retta e animata dall'idea di infinità che è in grado di ricomprendere tutte le idee della finitezza: infatti, la scienza mette a contatto con l'idea di infinito, che può trovare applicazione ed esemplificazione in ambiti finiti diversi ${ }^{40}$.

Secondo Husserl, a questo livello si situa la giustificazione di un impegno morale e sorge la coscienza della responsabilità della ragione ovvero la coscienza etica: «[...] l'uomo che già vive nella coscienza del potere della ragione si sa per questo responsabile per il giusto e l'ingiusto in tutte le sue attività, siano esse attività conoscitive, valutative o azioni volte ad avere un effetto reale» ${ }^{41}$. L'ideale dell'autentica humanitas sta allora nell'ideale dell'uomo razionale, non nel senso che abbia la facoltà della ragione, ma poi non fondi il suo agire sulla base di evidenze razionali, sibbene in quanto in ogni atto della sua vita opera in questo modo e aspira «[...] a ciò che dal punto di vista pratico è razionale in generale e puramente in virtù del suo assoluto valore

\footnotetext{
${ }^{38}$ HUSSERL, Lidea di Europa, p. 13-14.

${ }^{39}$ HUSSERL, L'idea di Europa, p. 17.

${ }^{40}$ HUSSERL, La crisi delle scienze europee e la fenomenologia trascendentale, p. 337.

${ }^{41}$ HUSSERL, L'idea di Europa, p. 38.
} 
pratico, e di conseguenza è impegnato coerentemente a conoscere in maniera evidente, e pertanto a realizzare, il vero e il bene pratici come il meglio della sua sfera pratica attuale ${ }^{42}$. Se questo ideale viene spinto al suo limite assoluto, costituisce l'ideale di una persona in quanto soggetto dell'assoluta ragione e forma il polo trascendente ogni finitezza, al quale ogni autentica aspirazione umana è rivolta, cioè l'idea di Dio. Su questa base non si fonda solo un'etica individuale, ma pure un'etica sociale, come scienza normativa dell'umanità che orienta la propria vita solo secondo norme di ragione ${ }^{43}$.

Husserl individua quindi la radice di ogni trasformazione e di ogni rinnovamento per l'Europa, a partire dalle rovine della guerra, all'interno del suo più ampio percorso di pensiero: la possibilità per la coscienza individuale e sociale di agire solo sulla base di motivazioni autenticamente razionali, in cui l'evidenza dell'apprensione delle essenze costituisce il telos cui l'umanità tende nella storia, in un cammino senza fine di crescita attraverso la radicale riflessione su di sé.

LENOCI, Michele. Crisis and revival in Europe: reflections upon phenomenological debate. Trans/Form/Ação, Marília, v. 37, p. 219-244, 2014. Edição Especial.

ABSTRACT: Through an analysis of works of Husserl and Scheler, this essay intends to examine their point of view regarding Europe and the European cultural tradition. It points out the evolution and development in their thought before and after the First World War.

KEYWORDS: Europe and philosophy. Phenomenology. Husserl. Scheler. First World War.

\section{Bibliografia}

CANFORA, L. 1914. Palermo: Sellerio, 2014.

FISCHER, F. Assalto al potere mondiale: la Germania nella guerra 1914-1918. Torino: Einaudi, 1965.

HUSSERL, E. Die Krisis der europäischen Menschentums und die Philosophie. In: . Die Krisis der europäischen Wissenschaften und die transzendentale Phänomenologie. A cura di W. Biemel. Den Haag: M. Nijhoff, 1954. p. 314-348. (Husserliana, v. 6). Tr. it. di E. Filippini, La crisi dell'umanità europea e la filosofia. In: La crisi delle scienze europee e la fenomenologia trascendentale. Milano: Il Saggiatore, 1961, p.328-358; pure in: Crisi e rinascita della cultura europea, p. 47-92.

\footnotetext{
${ }^{42}$ HUSSERL, L'idea di Europa, p. 40.

${ }^{43}$ HUSSERL, Lidea di Europa, p. 103.
} 
. Die Idee einer philosophischen Kultur. In:In: Erste Philosophie. A cura di R. Boehm. Den Haag: M. Nijhoff, 1956. p. 8-17; 203-207. (Husserliana, v. 7). Tr. it. di R. Cristin, Crisi e rinascita della cultura europea. Venezia: Marsilio, 1999, p. 29-44.

. Fichtes Menschheitsideal. In: Aufsätze und Vorträge 1911-21. A cura di Th. Nenon e H. R. Sepp. The Hague: M. Nijhoff, 1986. p. 267-293. (Husserliana, v. 25). Tr. it. di F. Rocci, Fichte e l'ideale di umanità. Tre lezioni. Pisa: Edizioni ETS, 2006.

. Aufsätze und Vorträge (1922-1937)., a cura di Th. Nenon e H.R. Sepp. Dordrecht: Kluwer, 1989. p. 3-94. (Husserliana, v. 27). Tr. it. Di C. Sinigaglia, L'idea di Europa. Milano: Cortina, 1999, p. 3-110.

MANN, Th. Pensieri di guerra. In: . Scritti storici e politici. Milano: Mondadori, 1957. Federico e la grande coalizione. Roma: Treves, 2006.

MEINECKE, F. Preußen und Deutschland im 19. und 20. Jahrhundert (1918). In: KESSEL, E. (Hrsg.). Brandenburg, Preußen, Deutschland. Kleine Schriften zur Geschichte und Politik, Werke. Stuttgart: Koehler, 1979. v. 9

SCHELER, M. Der Formalismus in der Ethik und die materiale Wertethik. Bern: Francke, 1966. p. 533. (Gesammelte Werke, v. 2). Tr. it. di G. Caronello, Il formalismo nell'etica e l'etica materiale dei valori. Cinisello Balsamo: Edizioni San Paolo, 1996.

Der Genius des Krieges und der deutsche Krieg (1915). In: Politischpädagogische Schriften. Francke Verlag, Bern 1982. p. 7-250. (Gesammelte Werke, v. 4)

Europa und der Krieg (1915). In: Politisch-pädagogische Schriften. Francke Verlag, Bern 1982. p. 251-266. (Gesammelte Werke, v. 4)

. Der Krieg als Gesamterlebnis (1916). In: Politisch-pädagogische Schriften. Francke Verlag, Bern 1982. p. 267-282. (Gesammelte Werke, v. 4)

Die Ursachen des Deutschenhasses (1917). In: Schriften. Francke Verlag, Bern 1982. p. 283-372. (Gesammelte Werke, v. 4)

Die christliche Liebesidee und die gegenwärtige Welt (1917). In: Vom Ewige im Menschen. Bonn: Bouvier, 2000. p. 357-401. (Gesammelte Werke, v. 5). Tr. it. di P. Premoli De Marchi: L'eterno nell'uomo. Milano: Bompiani, 2009, p. 881-981.

. Vom kulturellen Wiederaufbau Europas (1917). In: Vom Ewige im Menschen. Bonn: Bouvier, 2000. p. 405-447. (Gesammelte Werke, v. 5). Tr. it. di P. Premoli De Marchi: L'eterno nell'uomo. Milano: Bompiani, 2009, p. 985-1081.

Reue und Wiedergeburt (1917). In: Vom Ewige im Menschen. Bonn: Bouvier, 2000. p. 29-59. (Gesammelte Werke, v. 5). Tr. it. di P. Premoli De Marchi: L'eterno nell'uomo. Milano: Bompiani, 2009, p. 153-221.

. Der allgemeine Begriff von "Nation" und die konkreten Nationalideen (circa 1916). In: . Schriften zur Soziologie und Weltanschauungslehre. Bern: Francke, 1963. p. 334-347. (Gesammelte Werke, v. 5)

. Die Idee des Friedens und der Pazifismus (1931). In: Schriften aus dem Nachlaß. Bern: Francke, 1974. v. 4: Philosophie und Geschichte, p. 77-121. (Gesammelte Werke, v. 13). 